\title{
SELF EVALUATION PROGRAMME
}

ThE CANADIAN ANAESTHETISTS' SOCTETY SELF EVALUATION PROGRAMME will be available for the first time in September, 1972. This Programme consists of two hundred multiple choice questions. The emphasis will be on clinical problems, clinical physiology and clinical pharmacology. These questions may be done at your leisure with or without the aid of texts and journals. The Committee hopes that those participating in the Programme will complete two answer papers. One will be kept by the participant and the other forwarded to the Canadian Anaesthetists' Society, 178 St. George Street, Toronto 5, Ontario. Approximately six weeks after the release of the Programme, answer sheets with references will be mailed to the subscribers. The participants will remain completely anonymous, as the questions will be marked by a computer and the results will appear at a later date in the President's Newsletter.

The Education Committee of the Canadian Anaesthetists' Society hopes that you will participate in this event. A limited number of Programmes will be available. Register now to avoid later disappointment! Send requests for enrolment together with Registration Fee of $\$ 10.00$ to

\author{
Education Committee \\ Canadian Anaesthetists' Society \\ 178 St. George Street \\ Toronto 5, Ontario
}

\section{PROGRAMME D'AUTO-EVALUATION}

Le programme d'auto-évaluation de la Socitété Canadienne des Anesthésistes va être disponible vers le premier septembre 1972. Ce programme consiste en deux cents questions à choix multiples. On insiste surtout sur les problèmes cliniques, la pharmacologie et la physiologie cliniques. Vous pouvez répondre à ces questions dans vos temps libres avec ou sans l'aide de livres et de journaux. Les mernbres du comité espèrent que ceux qui participeront à ce programme consentiront à donner leurs réponses en double. Une copie sera conservée par l'auteur et l'autre sera adressée à la Société Canadienne des Anesthésistes, 178 rue SaintGeorge, Toronto 5, Ontario. Environ six semaines après le lancement du programme, les listes des réponses avec bibliographie seront adressées aux souscripteurs. Le plus complet anonymat sera assuré aux participants car les questions et réponses seront corrigées par computer et les résultats de la correction seront publiés plus tard dans le bulletin de nouvelles du président.

Les membres du comité d'éducation espèrent bien que vous prendrez part à cette évaluation personnelle. Nous n'avons qu'un nombre limité de programmes. Faites application dès maintenant afin de vous éviter des déceptions plus tard! Adressez vote demande de participation en même temps que le montant de votre cotisation qui est fixé à $\$ 10,00$.

\author{
A: Comité d'éducation \\ Société Canadienne des Anesthésistes \\ 170 rue Saint-George \\ Toronto 5, Ontario.
}

